\title{
Assessment of sarcopenia in patients with fibromyalgia
}

\author{
Abeline Kapuczinski $^{1}$ - Muhammad S. Soyfoo ${ }^{1} \cdot$ Sandra De Breucker ${ }^{2} \cdot$ Joëlle Margaux $^{1}$
}

Received: 22 June 2021 / Accepted: 14 August 2021 / Published online: 21 August 2021

(c) The Author(s) 2021

\begin{abstract}
Fibromyalgia is a chronic disorder characterized by persistent widespread musculoskeletal pain. Patients with fibromyalgia have reduced physical activity and increased sedentary rate. The age-associated reduction of skeletal muscle mass and function is called sarcopenia. The European Working Group on Sarcopenia in Older People developed a practical clinical definition and consensus diagnostic criteria for sarcopenia. Loss of muscle function is common in fibromyalgia and in the elderly. The goal of this study is to determine whether the reduction of muscle function in fibromyalgia is related to sarcopenia according to the European Working Group on Sarcopenia in Older People criteria. Forty-five patients with fibromyalgia and thirty-nine healthy control female subjects were included. All the participants were assessed by Fibromyalgia Impact Questionnaire and SARC-F questionnaire. Muscle mass was evaluated by bioimpedance analysis, muscle strength by handgrip strength test and physical performance with the Short Physical Performance Battery. Fibromyalgia Impact Questionnaire and SARC-F scores were statistically significantly higher in the fibromyalgia group than in the control group, showing severe disease and a higher risk of sarcopenia in the fibromyalgia group $(p<0.001)$. Muscle strength and physical performance were statistically significantly lower in the group with fibromyalgia than in the control group $(p<0.001)$. There was no statistical difference between fibromyalgia and control groups regarding skeletal muscle mass $(p=0.263)$. Our study demonstrated a significant reduction in muscle function in fibromyalgia patients without any loss of muscle mass. Loss of muscle function without decrease in muscle mass is called dynapenia.
\end{abstract}

Keywords Fibromyalgia $\cdot$ Muscle mass $\cdot$ Muscle strength $\cdot$ Physical performance $\cdot$ Sarcopenia

\section{Introduction}

Fibromyalgia (FM) is a chronic disorder characterized by persistent widespread musculoskeletal pain. It is associated with fatigue, sleep and cognitive disorders, anxiety and depression, leading to a poor quality of life [1]. It affects $2 \%$ of the general population and more particularly women $[2,3]$. The pathophysiology of FM is complex with several intricating factors. One of the main tenants underlying the disease is a somatosensory disturbance resulting in hypersensitization to pain (allodynia and hyperalgesia) [4]. The diagnosis of FM is based according to the 1990 and 2010

Abeline Kapuczinski

abeline.k@gmail.com

1 Department of Rheumatology, Hôpital Erasme, Université Libre de Bruxelles, 808, Route de Lennik, 1070 Bruxelles, Belgium

2 Department of Geriatrics, Hôpital Erasme, Université Libre de Bruxelles, Bruxelles, Belgium
American College of Rheumatology (ACR) diagnostic criteria [5, 6] as well as the updated criteria from 2016 [7]. There is currently no specific treatment of FM, the mainstay of treatment consisting primarily of pain management and physical rehabilitation.

As such, due to widespread pain, patients with FM have a reduced physical activity and a higher sedentary rate [8]. There are several converging lines of evidence demonstrating a significant reduction of muscular mass and physical activity and performance in patients with FM when compared with healthy controls matched for age and sex $[9,10]$. Furthermore, FM patients have a significantly increased muscular fat proportion compared to controls $[11,12]$. The resulting loss of muscular mass also known as sarcopenia is associated with an increased risk of falls and hence bone fractures [13].

In 2010, the European Working Group On Sarcopenia in Older People (EWGSOP) published a consensus definition of sarcopenia with diagnostic criteria for clinical practice [14]. These criteria were defined by including muscular 
mass as well as muscular function, strength and physical performance. In 2018, these criteria were updated with the definition of loss of muscular strength as the primary feature of sarcopenia. Sarcopenia was defined as probable if there was a loss of muscle strength and confirmed if the loss of strength was associated with reduced muscle mass. When poor physical performance is also detected, sarcopenia is considered severe [15].

Some studies have compared the physical function and condition of patients with FM to those of elderly patients and have showed that the loss of muscular strength and physical performance were not different $[10,16]$. Besides this, there was an increased risk of falls and loss of autonomy in FM patients similarly to that described in older population [13].

The aim of this study was to determine whether the reduction of muscle function in FM is related to sarcopenia according to the EWGSOP 2010 criteria.

\section{Patients and methods}

\section{Population}

The study was approved by the Ethics Committee of Erasme Hospital, with the reference SRB_201710_067 in January 2018. All the participants were informed about the objectives of the study and an informed consent was obtained. This study included two groups: a fibromyalgia group and a healthy control group. The recruitment of fibromyalgia subjects was done from the outpatient clinic from the Department of Rheumatology at Erasme Hospital. The healthy control group consisted of subjects among health care workers and other medical staff from Erasme Hospital on a voluntary basis. The inclusion criteria were female subjects, aged between 30 and 60 years and with a body mass index $(\mathrm{BMI}) \leq 30 \mathrm{~kg} / \mathrm{m}^{2}$. Pregnant women and patients carrying a pacemaker (which are contraindications for using a bioelectrical impedance analysis) were excluded from the study.

Forty-five patients with fibromyalgia, according to the 2010 ACR criteria, were included as well as 39 healthy control females. Patients in FM group did not have other chronic diseases nor other rheumatologic diseases. Anthropometrics measures (age, height, weight and BMI) of the patients were determined. We used the same measuring rod and the same scale for each evaluation. The patients were weighted with an empty stomach, preferably in the morning, with clothes and without shoes.

\section{Questionnaires}

Each patient or control filled the Fibromyalgia Impact Questionnaire (FIQ) of the fibromyalgia and the SARC-F questionnaire of the sarcopenia.
The FIQ assesses the impact of fibromyalgia on patients' activities of daily life, pain, fatigue, anxiety, depression [17]. It establishes a total score of 100 points, determining severe $(>59 / 100)$ or moderate (between 39 and 59/100) disease. The SARC-F questionnaire was developed to screen sarcopenia [18]. It includes five questions about strength, walking ability, stair climbing, rising from a chair and history of falls, giving a score ranging from 0 to 10 . A score above or equal to 4 points is predictive of sarcopenia [19].

\section{Assessment of muscle mass}

Muscle mass was quantified with a bio-electrical impedance analysis (BIA) which gives the volume of fat and lean body mass based on the relationship between the volume of a conductor and its electrical resistance [18]. We used the Bodystat QuadScan 4000 (Bodystat ltd, UK) to assess the fat mass content $(\mathrm{kg})$, the lean mass $(\mathrm{kg})$ and resistance at $50 \mathrm{kHz}(\mathrm{Ohms})$. The predicted skeletal muscle mass (SMM) was calculated using the equation of Janssen for the BIA [20]:

$$
\begin{aligned}
S M M= & \left(\text { height }^{2} / \text { BIA resistance } * 0.401\right)+(\text { gender } * 3.825) \\
& +(\text { age } *-0.071)+5.102), \text { with height in centimeters }, \\
& \text { BIA resistance in Ohms, gender } \\
= & 1 \text { for male and } 0 \text { for female, age in years. }
\end{aligned}
$$

Skeletal muscle mass index (SMI) was obtained by dividing SMM by height squared $\left(\mathrm{m}^{2}\right)$. We compared the results in the two groups with cut-offs defined by the EWGSOP where low muscle mass assessed by BIA is defined by a SMI below $6.42 \mathrm{~kg} / \mathrm{m}^{2}$ for women.

\section{Assessment of muscle strength}

Muscle strength was evaluated by a handgrip strength test with the Jamar dynamometer. Three measures for each arm have been taken and the best result for the dominant hand has been used for our study. Low muscle strength is characterized by a handgrip strength test below $20 \mathrm{~kg}$ for women.

\section{Assessment of physical performance}

Physical performance was assessed with the Short Physical Performance Battery (SPPB). It is a composite test of usual gait speed (over $4 \mathrm{~m}$ ), a balance test and a chair stand test. The scores range from 0 to 12 points: low performance $(0-6$ points), intermediate (7-9 points), high performance (10-12 points) [21]. 


\section{Assessment of sarcopenia}

The European Working Group on Sarcopenia in Older People (EWGSOP) developed in 2010 a practical clinical definition and consensus diagnostic criteria for sarcopenia with threshold values for geriatric populations. We used those values to assess all the participants for the muscle mass, muscle strength and physical performance [14]. According to the EWGSOP, the cut-off for sarcopenia is defined by values of predicted muscle mass lower than $6,42 \mathrm{~kg} / \mathrm{m}^{2}$. A low muscle strength was considered when the grip strength test was below $20 \mathrm{~kg}$ and a low physical performance when the SPPB score was below 8 points.

\section{Statistics}

One propensity score will be performed on two groups: Fibromyalgia $(n=45)$ and Control $(n=39)$. The CBPS R package will be used to perform the propensity score, estimating an Average Treatment Effect (ATE), using covariate balancing and requesting an exact match, which has been showed to be superior to traditional logistic regression approaches and boosted classification and regression trees [22]. An absolute standardized difference less than 10-15\% will be considered to support the assumption of balance between the groups because it is not affected by the sample size, unlike P-values, and it may be used to compare the relative balance of variables measured in different units [23]. The mean and standard deviation obtained after matching for continuous variables will be presented. After the propensity score, we will use the survey $\mathrm{R}$ package to perform linear regressions for continuous outcomes, which will include the treatment group effect, the weight resulting from the matching and variables present in the propensity score to obtain a doubly robust estimator which will correct the last remaining possible imbalance between the covariates and produces an unbiased treatment effect [29]. The survey R package includes the Huber-White-corrected standard errors, which maintains the standard errors unbiased even under heterogeneity of the residuals [30]. Last, the advantage of a doubly robust estimator is that it needs only one of the two models (propensity score and linear regression after the propensity score) to be correctly specified. The R software (R Core Team, 2017), version 3.4.3. was used to produce the results.

Before drawing conclusions on the above table, we have to apply a Bonferroni correction for multiple comparison purposes and we have to divide the p-value $(0.05)$ by the number of comparisons [9], that is, $0.05 / 9=0.0056$, to get the final $\mathrm{p}$-value on which we can draw conclusion. That is, any p-value below 0.0056 will be considered as significant: the next variables are significantly different between the two groups: FIQ, SARC-F, handgrip strength test and SPPB score.

\section{Results}

\section{Demographic characteristics}

Forty-five women with fibromyalgia and 39 healthy control female subjects were included in our study. Their demographic and clinical characteristics are represented in Table 1.

\section{Assessment of sarcopenia in the different study groups}

Table 2 shows the variables to assess sarcopenia for the two groups.

\section{FIQ and SARC-F questionnaires}

The mean value of the FIQ questionnaire for the group with fibromyalgia $(73 \pm 13)$ is significantly higher than that of the control group $(15 \pm 13)(p<0.001)$, displaying severe disease.

The FIQ value is not correlated with the physical performance (coefficient correlation $=-0.28, p=0.062$ ), nor with the muscle strength (coefficient correlation $=-0.23$, $p=0.125$ ). Therefore, the severity of the disease is not correlated with the muscle function.

The mean value of the SARC-F questionnaire was significantly higher $(5 \pm 2)$ in the fibromyalgia group compared to the control group $(0 \pm 0)(p<0.001)$. SARC-F questionnaire is predictive of sarcopenia in patients with fibromyalgia.

\section{Muscle mass}

According to the EWGSOP, low muscle mass with the BIA for women is defined by SMI $<6.42 \mathrm{~kg} / \mathrm{m}^{2}$. We did not observe any statistical difference between fibromyalgia and control groups regarding skeletal muscle mass $(p=0.263)$. There was no statistical difference for the fat mass $(p=0.217)$, lean mass $(p=0.304)$ and the SMM $(p=0.260)$.

Table 1 Demographic and clinical characteristics of the population including two groups, fibromyalgia and controls, represented by means \pm standard deviation and adjusted $p$ value

\begin{tabular}{lccc}
\hline & $\begin{array}{l}\text { Fibromyalgia, } \\
N=45\end{array}$ & Controls, $N=39$ & Adjusted $p$ value \\
\hline Age (years) & $48.86 \pm 8.66$ & $44.35 \pm 7.29$ & $\mathbf{0 . 0 1 3}$ \\
Weight $(\mathrm{kg})$ & $68.88 \pm 10.86$ & $62.92 \pm 11.83$ & $\mathbf{0 . 0 1 9}$ \\
Height $(\mathrm{m})$ & $1.61 \pm 0.06$ & $1.64 \pm 0.06$ & 0.06 \\
BMI $\left(\mathrm{kg} / \mathrm{m}^{2}\right)$ & $26.24 \pm 3.26$ & $23.13 \pm 3.53$ & $<\mathbf{0 . 0 0 1}$ \\
\hline
\end{tabular}

$B M I$ Body mass index 
Table 2 Variables used to assess sarcopenia in the two groups, with means \pm standard deviation and adjusted $p$ value

\begin{tabular}{lccc}
\hline Variable & Fibromyalgia $(n=45)$ & Control $(n=39)$ & Adjusted $p$ value \\
\hline FIQ $(/ 100)$ & $74 \pm 13$ & $15 \pm 13$ & $<\mathbf{0 . 0 0 1}$ \\
SARC-F $(/ 10)$ & $5 \pm 2$ & $0 \pm 0$ & $<\mathbf{0 . 0 0 1}$ \\
Fat mass $(\mathrm{kg})$ & $23.8 \pm 7.1$ & $23.4 \pm 7.7$ & 0.217 \\
Lean mass $(\mathrm{kg})$ & $42.4 \pm 6.3$ & $42.8 \pm 6.4$ & 0.304 \\
SMM $(\mathrm{kg})$ & $19.2 \pm 2.7$ & $19.6 \pm 2.8$ & 0.260 \\
SMI $\left(\mathrm{kg} / \mathrm{m}^{2}\right)$ & $7.2 \pm 0.5$ & $7.4 \pm 0.7$ & 0.263 \\
Handgrip strength test $(\mathrm{kg})$ & $18 \pm 8$ & $30 \pm 6$ & $<\mathbf{0 . 0 0 1}$ \\
SPPB score $(/ 12)$ & $8 \pm 2$ & $12 \pm 0$ & $<\mathbf{0 . 0 0 1}$ \\
\hline
\end{tabular}

SMM skeletal muscle mass, SMI skeletal muscle mass index, SPPB Short Physical Performance Battery

\section{Muscle strength}

The cut-off value for the dominant hand in women, according to the EWGSOP, is below $20 \mathrm{~kg}$. There was a statistically significant loss of muscle strength in fibromyalgia group $(18 \pm 8)$ compared to the control group $(30 \pm 6)(p<0.001)$.

\section{Physical performance}

A SPPB score below 8 signs a loss of physical performance. Patients with fibromyalgia had a statistically significant lower SPPB score $(8 \pm 2)$ compared to control groups $(12 \pm 0)(p<0.001)$. This shows that fibromyalgia patients have a loss of physical performance relative to controls.

\section{Discussion}

The goal of this study was to determine whether the reduction of muscle function in fibromyalgia is related to sarcopenia according to the EWGSOP 2010 criteria. We have identified a loss of muscle function in fibromyalgia (loss of muscle strength and physical performance), but there was no loss of muscle mass, the key feature in sarcopenia.

Muscle mass was studied with a bio-electrical impedance analysis (BIA). Other methods are also used in clinical practice. For example, Magnetic Resonance Imaging (MRI) or the CT-scan are used for the non-invasive assessment of muscle mass. Dual-energy x-ray absorptiometry (DXA) is an alternative method for distinguishing the fat mass, the lean mass and bone density [24]. We chose the BIA because this method is not expensive and easy to use in daily practice [14]. The disadvantage of the BIA is that it overestimates muscle mass and underestimates fat mass [18]. Koca et al. assessed patients with fibromyalgia in terms of sarcopenia using BIA, anthropometrics measures, a handgrip strength test and a gait speed test over $6 \mathrm{~m}$. Muscle strength and gait speed were lower in patients with fibromyalgia. The body composition according to the BIA and anthropometrics measures were not significantly different between the two groups [25].

The contribution of our study in relation to Koca's work was to use the EWGSOP 2010 criteria and their threshold values for each component of sarcopenia. Physical performance was evaluated with different tests including a gait speed, a balance test and a chair stand test. Furthermore, a screening test of sarcopenia was realized, using the SARC-F questionnaire. Furthermore, we limited the age of all participants between 30 and 60 years because of the impending loss of muscle mass after the third decade [26]. BMI $<30 \mathrm{~kg} /$ $\mathrm{m}^{2}$ was included to uniform the two groups for the muscle mass, and to avoid the bias of significantly higher fat mass in obese patients that could warp the interpretation of the results.

Muscle mass of patients with fibromyalgia has been studied in different works and did not show any difference compared to healthy people, as assessed by BIA [11, 16, 25]. However, some studies showed a difference in body composition in terms of fat mass and lean mass, showing that women with fibromyalgia had more fat mass than healthy controls $[11,12]$. In our study, we did not observe that body fat composition was different between the two study groups.

Regarding muscle function, we also observed similarly to other studies that patients with fibromyalgia exhibit a loss of muscle strength and physical performance [8-10, 13]. Loss of muscle function without loss of muscle mass is named dynapenia. It is defined as the age-related loss of muscle strength and it is often confused with sarcopenia [27]. Sarcopenia is the atrophy of muscular fibers associated with the reduction of the number of the fibers, while dynapenia is a muscular atrophy with a conserved number of fibers responsible for a loss of muscle strength without loss of muscle mass. Based on our results, we can define patients with fibromyalgia as suffering from dynapenia.

In early 2018, the EWGSOP updated the definition of sarcopenia with new recommendations. A low muscle strength is now considered to be the primary indicator of probable sarcopenia. When a low muscle strength is detected, a sarcopenia diagnosis must be confirmed by the 
presence of a concomitant low muscle mass. The severity of the disease is evaluated with the study of physical performance [15].

In clinical practice, according to the new recommendations in 2018 by the EWGSOP, the SARC-F questionnaire helps to find patients at risk of sarcopenia. A SARC-F score $\geq 4 / 10$ is an indication to measure muscle strength. Low muscle strength is predictive of probable sarcopenia that must be confirmed with the measure of low muscle mass. Low physical performance with a SPPB score $\leq 8 / 12$ indicates the severity of the sarcopenia. According to those criteria, sarcopenia is now considered as a muscle disease. Otherwise, cut-off points have been reviewed for muscle strength ( $<16 \mathrm{~kg}$ for women) and data are available for men and women according to their centiles. If we apply the new recommendations, the SARC-F questionnaire is predictive of sarcopenia in our Fibromyalgia population. The mean muscle strength in this group is $18 \mathrm{~kg}$, but according to the mean age of 48 years old, we are under the 10th centile, defining low muscle strength and probable sarcopenia. As muscle mass was normal using the BIA, which is known to overestimate muscle mass, we cannot confirm the disease.

Some limitations should be considered in our study. First, we did not consider the sedentary lifestyle of the controls, the hormonal or the nutritional status, nor comorbidities. Moreover, alcohol consumption, smoking or drug uses were not taken into account in our study. Second, assessment of sarcopenia was performed during a single visit and not repeatedly over a time span for more accurate determination of muscle mass loss.

Finally, we decided to consider only women in our study because FM is mostly a women's disorder [28].

\section{Conclusions}

There is no sarcopenia in patients with fibromyalgia according to the original definition of the EWGSOP in 2010, but there is a possible sarcopenia with the 2018 updates of the algorithm. Our study demonstrated a significant reduction in muscle function in fibromyalgia patients (decreased in muscle strength and in physical performance) without any loss of muscle mass. Loss of muscle function without decrease in muscle mass is named dynapenia. Screening to detect and prevent sarcopenia should be conducted in patients with fibromyalgia as loss of muscle function is common in these patients.

Supplementary Information The online version contains supplementary material available at https://doi.org/10.1007/s00296-021-04973-6.
Acknowledgements The authors would like to thank Jean-François Fils for his help with the statistics part of this study and Pr Valérie Gangji for her help in the study as well.

\section{Declarations}

Conflict of interest The authors declare no potential financial or personal conflict of interest.

Open Access This article is licensed under a Creative Commons Attribution 4.0 International License, which permits use, sharing, adaptation, distribution and reproduction in any medium or format, as long as you give appropriate credit to the original author(s) and the source, provide a link to the Creative Commons licence, and indicate if changes were made. The images or other third party material in this article are included in the article's Creative Commons licence, unless indicated otherwise in a credit line to the material. If material is not included in the article's Creative Commons licence and your intended use is not permitted by statutory regulation or exceeds the permitted use, you will need to obtain permission directly from the copyright holder. To view a copy of this licence, visit http://creativecommons.org/licenses/by/4.0/.

\section{References}

1. Clauw DJ (2014) Fibromyalgia: a clinical review. JAMA 311(15):1547-1555

2. Macfarlane GJ, Kronisch C, Dean LE, Atzeni F, Häuser W, Fluß E et al (2017) EULAR revised recommendations for the management of fibromyalgia. Ann Rheum Dis 76(2):318-328

3. Clauw DJ, D'Arcy Y, Gebke K, Semel D, Pauer L, Jones KD (2018) Normalizing fibromyalgia as a chronic illness. Postgrad Med 130(1):9-18

4. Petersel DL, Dror V, Cheung R (2011) Central amplification and fibromyalgia: disorder of pain processing. J Neurosci Res 89(1):29-34

5. Wolfe F, Clauw DJ, Fitzcharles M-A, Goldenberg DL, Katz RS, Mease P et al (2010) The American College of Rheumatology preliminary diagnostic criteria for fibromyalgia and measurement of symptom severity. Arthritis Care Res 62(5):600-610

6. Wolfe F, Smythe HA, Yunus MB, Bennett RM, Bombardier C, Goldenberg DL et al (1990) The American College of Rheumatology 1990 criteria for the classification of fibromyalgia. Arthritis Rheum 33(2):160-172

7. Wolfe F, Clauw DJ, Fitzcharles M-A, Goldenberg DL, Häuser W, Katz RL et al (2016) 2016 Revisions to the 2010/2011 fibromyalgia diagnostic criteria. Semin Arthritis Rheum 46(3):319-329

8. Ángel P, Roman L, Campos MAS, e, Meza M, Armando J, Delgado Fernández M, et al (2012) Analysis of the physical capacity of women with fibromyalgia according to the severity level of the disease. Rev Bras Med Esporte 18(5):308-312

9. Maquet D, Croisier J-L, Renard C, Crielaard J-M (2002) Muscle performance in patients with fibromyalgia. Joint Bone Spine 69(3):293-299

10. Panton LB, Kingsley JD, Toole T, Cress ME, Abboud G, Sirithienthad $\mathrm{P}$ et al (2006) A comparison of physical functional performance and strength in women with fibromyalgia, age- and weightmatched controls, and older women who are healthy. Phys Ther 86(11):1479-1488

11. Segura-Jimenez V, Aparicio VA, Alvarez-Gallardo IC, CarbonellBaeza A, Tornero-Quinones I, Delgado-Fernandez M (2015) Does body composition differ between fibromyalgia patients and controls? The al-ándalus project. Clin Exp Rheumatol 33(1 Suppl 88):S25-32 
12. Lobo MM, Paiva ED, Andretta A, Schieferdecker ME (2014) Body composition by dual-energy X-ray absorptiometry in women with fibromyalgia. Rev Bras Reumatol 54(4):273-278

13. Góes SM, Leite N, Shay BL, Homann D, Stefanello JMF, Rodacki ALF (2012) Functional capacity, muscle strength and falls in women with fibromyalgia. Clin Biomech 27(6):578-583

14. Cruz-Jentoft AJ, Baeyens JP, Bauer JM, Boirie Y, Cederholm T, Landi F et al (2010) Sarcopenia: European consensus on definition and diagnosis: report of the European working group on sarcopenia in older people. Age Ageing 39(4):412-423

15. Cruz-Jentoft AJ, Bahat G, Bauer J, Boirie Y, Bruyère O, Cederholm $T$ et al (2018) Sarcopenia: revised European consensus on definition and diagnosis. Age Ageing. https://doi.org/10.1093/ ageing/afz046

16. Latorre-Román PÁ, Segura-Jiménez V, Aparicio VA, Campos MA, García-Pinillos F, Herrador-Colmenero M, Álvarez-Gallardo IC, Delgado-Fernández M (2015) Ageing influence in the evolution of strength and muscle mass in women with fibromyalgia: the al-Ándalus project. Rheumatol Int 35(7):1243-1250

17. Bennett R (2005) The fibromyalgia impact questionnaire (FIQ): a review of its development, current version, operating characteristics and uses. Clin Exp Rheumatol 23(5 Suppl 39):S154-162

18. Beaudart $\mathrm{C}, \mathrm{McCl}$ loskey $\mathrm{E}$, Bruyère $\mathrm{O}$, Cesari M, Rolland $\mathrm{Y}$, Rizzoli R et al (2016) Sarcopenia in daily practice: assessment and management. BMC Geriatr 16(1):170

19. Malmstrom TK, Morley JE (2013) SARC-F: a simple questionnaire to rapidly diagnose sarcopenia. J Am Med Dir Assoc 14(8):531-532

20. Janssen I, Heymsfield SB, Ross R (2002) Low relative skeletal muscle mass (sarcopenia) in older persons is associated with functional impairment and physical disability. J Am Geriatr Soc 50(5):889-896

21. Treacy D, Hassett L (2018) The short physical performance battery. J Physiother 64(1):61

22. Imai K, Ratkovic M (2015) Robust estimation of inverse probability weights for marginal structural models. J Am Stat Assoc 110(511):1013-1023
23. Austin PC (2011) An introduction to propensity score methods for reducing the effects of confounding in observational studies. Multivariate Behav Res 46(3):399-424

24. Beaudart C, Reginster J-Y, Slomian J, Buckinx F, Locquet M, Bruyère $O$ (2014) Prevalence of sarcopenia: the impact of different diagnostic cut-off limits. J Musculoskelet Neuronal Interact 14(4):425-431

25. Koca I, Savas E, Ozturk ZA, Boyaci A, Tutoglu A, Alkan $S$ et al (2016) The evaluation in terms of sarcopenia of patients with fibromyalgia syndrome. Wien Klin Wochenschr 128(21-22):816-821

26. Janssen I, Heymsfield SB, Wang ZM, Ross R (2000) Skeletal muscle mass and distribution in 468 men and women aged 18-88 yr. J Appl Physiol 89(1):81-88

27. Clark BC, Manini TM (2012) What is dynapenia? Nutrition 28(5):495-503

28. Wolfe F, Walitt B, Perrot S, Rasker JJ, Häuser W (2018) Fibromyalgia diagnosis and biased assessment: sex, prevalence and bias. PLoS ONE 13(9):e0203755

29. Funk MJ, Westreich D, Wiesen C, Stürmer T, Brookhart MA, Davidian M (2011) Doubly robust estimation of causal effects. Am J Epidemiol. 173(7):761-7. https://doi.org/10.1093/aje/kwq439. Epub 2011 Mar 8. PMID: 21385832; PMCID: PMC3070495. Accessed 1 Apr 2011

30. Winston Lin (2013) Agnostic notes on regression adjustments to experimental data: Reexamining Freedman's critique. The Annals of Applied Statistics, Ann Appl Stat. 7(1):295-318. https://doi. org/10.1214/12-AOAS583

Publisher's Note Springer Nature remains neutral with regard to jurisdictional claims in published maps and institutional affiliations. 